\title{
Adrenalin für das „Notfallset“
}

\section{Die FCKW-Halon-Verbots-Verordnung verbietet in $\S 2$ das Inverkehrbringen von FCKW-haltigen Druckgaspackungen. Davon betroffen sind auch Adrenalin-Dosieraerosole für "Notfallsets“. In begründeten Ausnahmefällen ist der Bezug aus dem Ausland jedoch rechtskonform.}

$\mathrm{P}$ atienten mit der Anamnese einer Anaphylaxie - insbesondere durch Insektengift, aber auch durch andere Auslöser - wird für die Initialtherapie erneuter Reaktionen ein "Notfallset“ zur Selbstmedikation verordnet. Empfohlener Bestandteil dieser Ausrüstung ist ein Adrenalinpräparat. $\mathrm{Ob}$ für das „Notfallset“ Adrenalin als Fertigspritze zur Injektion oder als Dosieraerosol zur Inhalation besser geeignet ist, wird kontrovers diskutiert (McLean-Tooke APC et al. BMJ 2003; 327: 1332-5). Im Allgemeinen wird ein EpinephrinDosieraerosol zuverlässiger angewandt, so dass Adrenalin zur Injektion nur in besonderen Fällen verordnet werden sollte (Tryba M et al. Allergo J 1994; 3: 211-24, Crifo G et al. Allergologie 2003; 26: 516-23).

Seit einigen Jahren ist in Deutschland kein für das „Notfallset“ geeignetes Adrenalin-Präparat mehr auf dem Markt. Daher wurde (und wird) Epinephrin-haltiges Dosieraerosol aus dem Ausland bezogen, in der letzten Zeit im Wesentlichen das US-amerikanische Produkt Primatene ${ }^{\circledR}$ Mist. Hierdurch wird aber gegen die FCKW-HalonVerbots-Verordnung verstoßen, da diese Präparate bestimmte FCKW als Treibmittel enthalten. In der Folge wurde unter anderem von der Bezirksregierung Braunschweig dargelegt, dass die Einfuhr von Primatene ${ }^{\circledR}$ Mist zur Versorgung lebensbedrohlich gefährdeter Patienten unzulässig sei und einen Straftatbestand darstelle (Allergo Journal 2003; 12: 210). Dies führte zu einer erheblichen Verunsicherung unter den Allergologen.

Nach aktuellem Wissensstand ist der Bezug FCKW-haltigen AdrenalinDosier-Aerosols aus dem Ausland jedoch unter bestimmten Voraussetzungen rechtskonform möglich. Das Baye- rische Staatsministerium für Umwelt, Gesundheit und Verbraucherschutz führte auf Anfrage hierzu unter anderem Folgendes aus: „Die für den Vollzug der Verbote des Inverkehrbringens bestimmter FCKW zuständigen Bayerischen Gewerbeaufsichtsämter wurden bereits in einem Schreiben aus dem Jahr 1998 angewiesen, gegen die Verwendung eines FCKW-haltigen Sprays nicht einzuschreiten, falls ein Arzt ein derartiges Medikament im Einzelfall für einen speziellen Patienten unter Inanspruchnahme der Vorschriften des $\$ 34$ StGB zur Behandlung einer besonderen Notafallsituation verschreibt,
Sehr geehrte Patientin,

sehr geehrter Patient, des Mundstückes. die Flasche mehrmals.

5. Atmen Sie tief aus. der ausatmen. Mundstück. wenn andere Medikamente zu diesem Zweck nicht geeignet sind. Der verschreibende Arzt trägt dabei die Verantwortung für die Inanspruchnahme des ,rechtfertigenden Notstands' nach $\$ 34$ StGB."

$\mathrm{Da}$ derzeit kein anderes für das "Notfallset" geeignetes Dosieraerosol auf dem deutschen Markt verfügbar ist, kann „zur Behandlung einer besonderen Notfallsituation" FCKW-haltiges Dosieraerosol verschrieben werden. Die Notwendigkeit ist sowohl in den Krankenunterlagen als auch auf dem Rezept zu vermerken. Hierzu reicht es, die Diagnose anzugeben und darauf hinzuweisen, dass es keine sinnvolle therapeutische Alternative gibt.

Da die Gebrauchsanleitung von Primatene ${ }^{\circledR}$ Mist in Englisch verfasst ist, finden Sie anbei einen Vorschlag für ein Patientenmerkblatt, in dem die Handhabung des Präparates kurz auf Deutsch erklärt ist.

Prof. Dr. Thomas Fuchs, Göttingen Prof. Dr. Bernhard Przybilla, München

\section{Anleitung zur Anwendung von Primatene ${ }^{\circledR}$ Mist}

zur Notfall-Behandlung haben wir Ihnen ein Spray namens „Primatene ${ }^{\circledR}$ Mist“ verschrieben. Dieses Präparat wird aus den USA importiert und enthält leider nur eine englische Gebrauchsanweisung. Nachfolgend möchten wir Ihnen den Gebrauch dieses Präparates kurz auf Deutsch erklären:

1. Nehmen Sie die Schutzkappe vom Mundstück ab.

2. Entfernen Sie das Plastikmundstück von der Flasche.

3. Stecken Sie den schmalen Metallhals der Flasche in die dünne Plastiköffnung

4. Nehmen Sie die Flasche zwischen Daumen und Zeigefinger und schütteln Sie

6. Nehmen Sie das Mundstück in den Mund und umschließen Sie es mit den Lippen. Während Sie langsam und gleichmäßig einatmen, drücken Sie mit Daumen und Zeigefinger Mundstück und Flasche zusammen. Dadurch entleert sich das Medikament in einem Sprühstoß. Der Wirkstoff muss unbedingt mit dem Einatmungsstrom tief in den Bronchialbereich gebracht werden.

7. Halten Sie den Atem möglichst lange (10 Sekunden) an, bevor Sie langsam wie-

8. Nach Benutzung des Präparates setzen Sie die Schutzkappe wieder auf das 